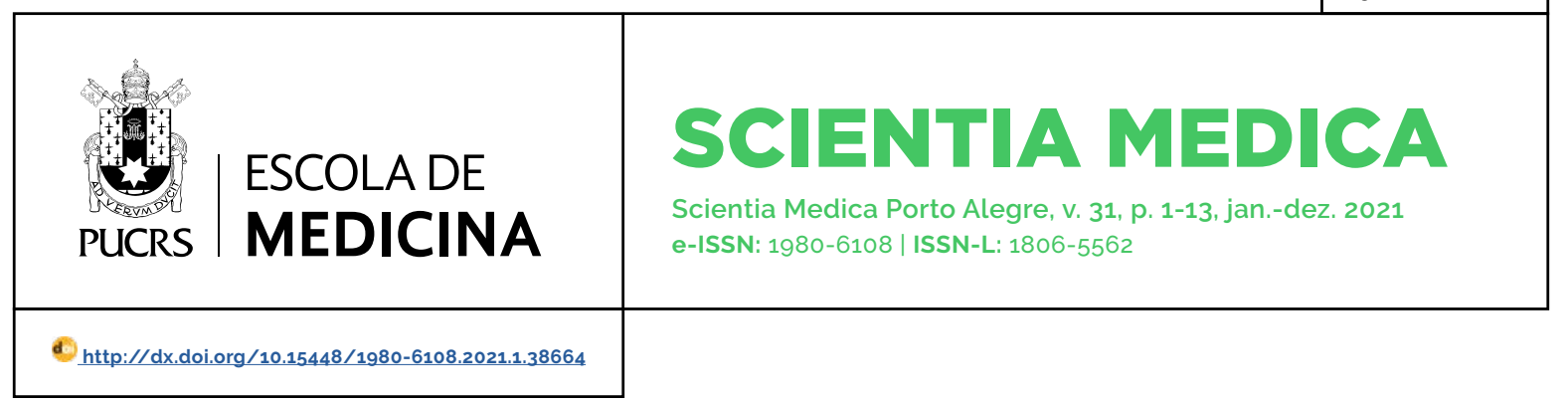

SEÇÃO: ARTIGO ORIGINAL

\title{
Associação da distância da habitação em relação a sítios de reciclagem sobre habilidades cognitivas em escolares
}

\author{
Association of housing distance in relation to recycling sites on schoolchildren's \\ cognitive abilities
}

Luciana Cristina Mancio
Balico $^{1}$

orcid.org/0000-0002-4918-3325

lcmbalico@ucs.br

\section{Diane Arbusti ${ }^{1}$}

orcid.org/0000-0002-7969-8290

dianearbusti@gmail.com

\section{Luciano da Silva \\ Selistre ${ }^{1}$}

orcid.org/0000-0002-0152-0636

Isselist@ucs.br

\section{Ana Maria Paim \\ Camardelo ${ }^{1}$}

orcid.org/0000-0001-7654-2058

ampcamar@ucs.br

\section{Emerson Rodrigues da} Silva ${ }^{1}$

orcid.org/0000-0001-5421-2883 ersilvag@ucs.br

Recebido em: 30 jul. 2020 Aceito em: 18 mar. 2021. Publicado em: 27 maio 2021

\section{(c) (1)}

Artigo está licenciado sob forma de uma licença Creative Commons Atribuição 4.0 Internacional.

\section{Resumo}

Objetivos: Verificar a associação da distância da habitação em relação a sítios de reciclagem sobre habilidades cognitivas em escolares.

Métodos: Estudo transversal, no qual aplicou-se a escala Wechsler Abreviada de Inteligência em 100 escolares de 6 a 14 anos. As crianças foram divididas em um grupo de estudo (expostos), cujas residências estavam situadas a menos de 100 metros de sítios de reciclagem, em um bairro da cidade de Caxias do Sul. Houve um grupo controle, cujas residências estavam a mais de 150 metros de algum sítio de reciclagem, no mesmo bairro ou adjacências.

Resultados: A habitação próxima a sítios de reciclagem aumentou a chance de apresentar quociente de inteligência baixo nos escolares na análise univariada (OR 2,19; IC95\% 1,21-3,95). No quociente de inteligência total, o escore obtido foi de 84 no grupo exposto e 95 no grupo controle $(p \leq 0,01)$. Quando ajustadas para outras variáveis potencialmente prejudiciais, a escolaridade materna elevada mostrou-se um fator atenuador do impacto da distância (OR 0,28; IC95\% 0,11-0,72). A diferença encontrada entre os escores de quociente de inteligência verbal foi de 14 pontos $(p<0,01)$; no quociente de inteligência de execução a diferença foi de 4 pontos $(p=0,04)$.

Conclusões: A distância entre a habitação e os sítios de reciclagem de resíduos pode reduzir habilidades cognitivas em escolares, mas esse efeito parece ser atenuado pela escolaridade materna. Essa associação reforça as preocupações sobre o impacto do manejo inadequado de residuos urbanos, podendo vir a subsidiar a criação de políticas públicas que visem diminuir exposições ambientais potencialmente danosas.

Palavras-chave: Crescimento e desenvolvimento, disfunção cognitiva, resíduos sólidos, testes de inteligência.

\section{Abstract}

Aims: We verified the association of housing distance in relation to recycling sites on schoolchildren's cognitive abilities.

Methods: This is a cross-sectional study, in which the Wechsler Abbreviated Scale of Intelligence was applied to 100 schoolchildren aged 6 to 14 years. Children were divided into a study group (exposed), whose households were located less than 100 meters from recycling sites in a neighborhood in the city of Caxias do Sul, and a control group, whose homes were located more than 150 meters from a recycling site in the same neighborhood or nearby.

Results: Residences located more than 150 meters away from a recycling site can reduce the chance of low intelligence quotient in schoolchildren in the univariate analysis (OR 2.19; $95 \% \mathrm{Cl}$ 1.21-3.95). The full-scale intelligence quotient score was 84 in the exposed group and 95 in the control group $(p \leq 0.01)$. When adjusted for other potentially harmful variables, higher maternal education was a mitigating factor of distance impact (OR $0.28,95 \% \mathrm{Cl} 0.11-0.72$ ). The difference 
found among verbal intelligence quotient scores was 14 points $(p<0.01)$; in the performance intelligence quotient, the difference was 4 points $(p=0.04)$.

Conclusions: Living close to waste recycling sites can reduces cognitive abilities in schoolchildren, and this effect seems to be attenuated by maternal education. This association reinforces concerns about the impact of inadequate urban waste management, and may subsidize the creation of public policies that aim to reduce potentially harmful environmental exposures.

Keywords: Cognitive dysfunction, solid waste, recycling, intelligence tests.

Abreviaturas: QI, quociente de inteligência; WASI, escala Wechsler Abreviada de Inteligência (Wechsler Abbreviated Scale of Intelligence).

\section{Introdução}

Em muitos países em desenvolvimento, os residuos urbanos são, inicialmente, processados dentro de comunidades, em terrenos contíguos aos locais onde crianças habitam, transitam e brincam. A população que reside próximo aos centros de reciclagem é desfavorecida no que se refere à saúde, habitação, educação, segurança e saneamento básico. Esses resíduos podem conter vários tipos de rejeitos, como resíduos orgânicos, plásticos, recipientes industriais, produtos químicos e até mesmo rejeitos de serviços de saúde. Pessoas que moram e/ou trabalham em centros de reciclagem estão expostas a muitos efeitos nocivos de poluentes, e as crianças são particularmente vulneráveis aos efeitos neurotóxicos desse contato (1-5). Pessoas mais jovens ingerem mais comida, ar e água, proporcionalmente ao próprio tamanho do que os adultos e, portanto, têm maiores exposições a produtos químicos tóxicos em proporção ao peso corporal. Além disso, as vias metabólicas são imaturas, assim como a barreira hemato-encefálica, que é mais permeável a compostos neurotóxicos, sem contar o fato de que crianças têm mais tempo de vida para desenvolver doenças crônicas, uma vez expostas a um poluente $(6,7)$. A exposição a niveis mais baixos de metais neurotóxicos (metilmercúrio, chumbo, bifenilos policlorados, pesticidas, arsênico, manganês, fluorido) está associada a problemas comportamentais (agressão, transtorno opositor desafiador, problemas de conduta) $(8,9)$; transtorno de déficit de atenção e hiperatividade
(8-10); efeitos adversos cardiovasculares (diminuição da frequência cardiaca, doença coronariana, cardiopatia congênita) (6), imunológicos (doenças infecciosas, câncer, asma, atopia) (6); e endócrinos (início tardio da puberdade, dislipidemia, alterações da homeostase da glicose, problemas na tireoide, aumento adiposo, baixo peso ao nascer, baixa estatura) $(1,11,12)$. Assim, a contaminação ambiental deve ser levada em consideração na avaliação do desenvolvimento neurocognitivo (9).

Até o presente momento, os autores têm conhecimento de que os estudos publicados até então avaliam sítios de descarte do chamado "lixo eletrônico" ou "e-waste" (10), ou exposições específicas como flúor (9), chumbo $(3,11)$, zinco (12), cadmio (10) e manganês (13). No entanto, a busca por um poluente específico, e a conseguinte correlação com desfechos em saúde pediátrica, pode ser limitada em virtude da variedade de conteúdo nos rejeitos. Alguns podem conter poluentes não pesquisados, assim como outros, investigados, podem não estar presentes na amostra e no local de estudo. Dessa forma, optou-se por uma abordagem da exposição (proximidade dos locais de reciclagem) ao invés da busca por apenas um poluente especifico (14). Além disso, desconhecem-se estudos avaliando o impacto da proximidade a lugares de reciclagem sobre o quociente de inteligência (QI) em população pediátrica de uma comunidade desfavorecida.

Considerando a ameaça à saúde, que representa a exposição aos resíduos urbanos, e a prática de processamento de resíduos em áreas onde brincam e vivem crianças em idade escolar, é fundamental investigar o impacto desses efeitos, pensando em um plano de manejo ambiental, clínico, psíquico e pedagógico. Este estudo procurou avaliar a associação entre a proximidade da residência em relação a sítios de reciclagem de residuos e a redução no QI de escolares, avaliada através de uma escala padronizada e validada no Brasil. Esses dados poderão fornecer importantes informações para a análise de risco ambiental gerado pelo processo de reciclagem inadequado e o consequente impacto no desenvolvimento cognitivo das crianças que vivem próximas a esses locais. 


\section{Métodos}

Foi realizado um estudo transversal, com crianças de 6 a 14 anos, que estavam regularmente matriculadas e frequentando a escola, moradoras de comunidades próximas a sítios de reciclagem de resíduos urbanos, na cidade de Caxias do Sul, Rio Grande do Sul. Dados perinatais, demográficos e de neurodesenvolvimento de 157 crianças dessa comunidade foram levantados em 2018 e descritos em um estudo prévio, do qual foram selecionados, aleatoriamente, uma amostra de 100 crianças, sendo 51 para o grupo de expostos e 49 para o grupo controle (15). O critério de alocação dessas crianças, tanto no estudo prévio quanto no presente estudo, foi a moradia localizada até 100 metros de distância de um sítio de reciclagem, aberto e sem divisórias protetoras ao acesso de pessoas. O grupo controle foi selecionado entre escolares do mesmo bairro, mas que tinham residência localizada a mais de 150 metros de distância do centro de uma reciclagem, permitindo uma zona de eliminação (wash out) entre os expostos e as crianças do grupo controle (Figura 1). Como critérios de exclusão, foram consideradas crianças com algum problema grave de neurodesenvolvimento conhecido.

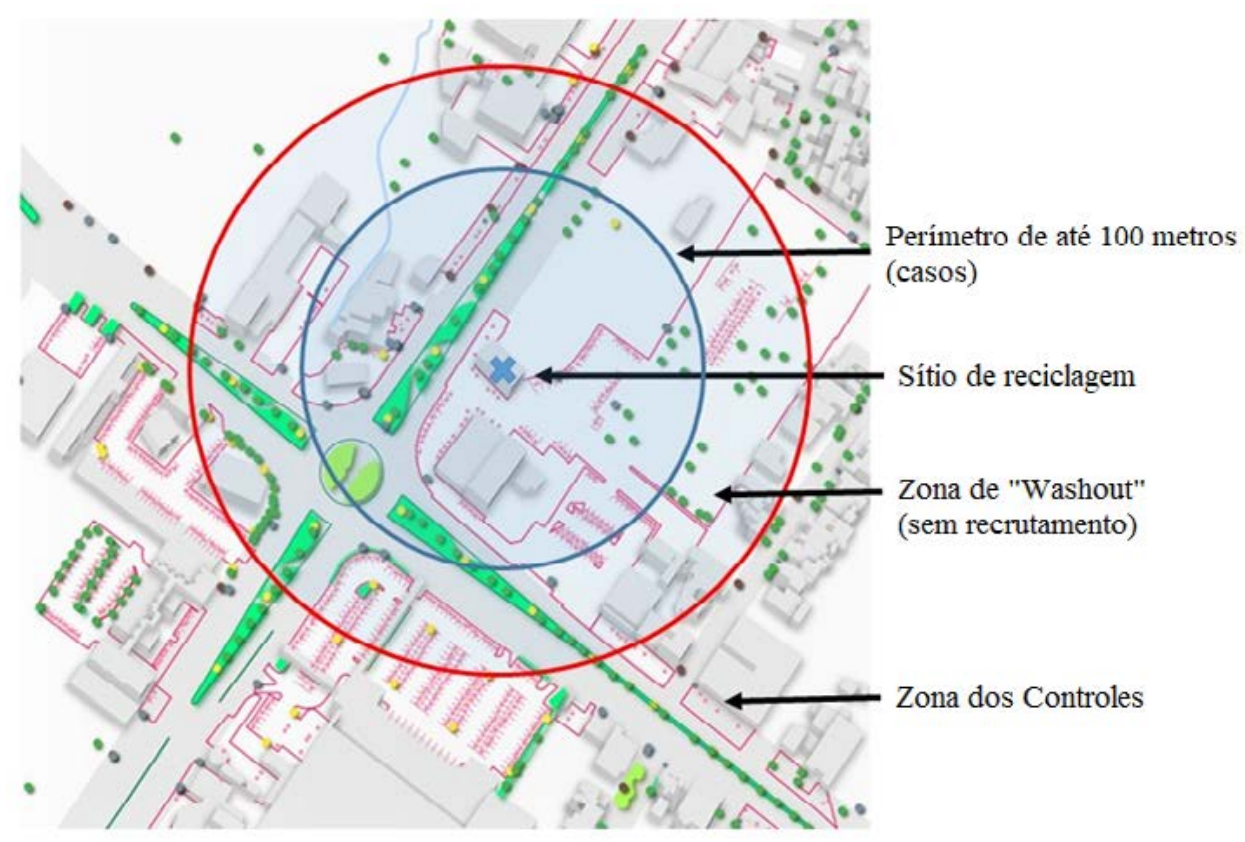

Figura 1 - Identificação geográfica dos sítios de reciclagem e a delimitação do grupo de expostos.

O bairro estudado localiza-se na cidade de Caxias do Sul, no sul do Brasil. No local em questão, os resíduos sólidos são tratados por cooperativas ou organizações familiares, que fazem a separação e destinação nos próprios locais de habitação ou em terrenos contíguos às residências. Esses residuos são oriundos tanto de residências quanto de indústrias e empresas da cidade.

A demarcação do ponto central do sítio de reciclagem e do local de habitação dos participantes da pesquisa foi feita por meio de georeferenciamento. Para a determinação dos pontos de habitação e reciclagem, usou-se o aplicativo Map
Plus (Miocool Inc.) versão 2.6.9, e para o cálculo das distâncias, o software Quantum Gis (QGIS) versão 2.14.20. O QGIS é um Sistema de Informação Geográfica, que permitiu identificar os dois grupos de escolares participantes da pesquisa (16).

O escore de QI foi obtido através da aplicação presencial da escala Wechsler Abreviada de Inteligência (WASI) (17), que é um instrumento usado mundialmente para avaliação da inteligência, aplicável a crianças de seis anos até idosos de 89 anos de idade, validado no Brasil desde 2014. A WASI foi aplicada individualmente, com duração aproximada de 30 minutos. 
As variáveis estudadas foram: QI execução, que é a capacidade fluida da inteligência, rapidez e exatidão do raciocínio abstrato, especialmente para resolução de novos problemas; QI verbal, que é a capacidade cristalizada da inteligência, conhecimento e vocabulário adquiridos; QI total, formado pelos QI execução e QI verbal.

Os quatro subtestes que compõem os QI são: vocabulário - que mede memória, habilidade de aprendizagem, conhecimento verbal e desenvolvimento de linguagem -; cubos - mede a percepção e análise visual de figuras abstratas, construção do todo a partir de partes, processo de informação visoespacial e de organização perceptual, coordenação visomotora, raciocínio espacial -; semelhanças - mede a formação de conceitos verbais, relaciona conceitos entre si e generaliza as relações existentes entre dois conceitos, raciocínio verbalabstrato -; raciocínio matricial - mede raciocinio fluido, capacidade de manipular mentalmente símbolos abstratos e perceber a ralação entre ambos. Para todas essas variáveis, os resultados são expressos como um escore de pontos derivado de um coeficiente do desvio de uma curva normal, com uma média de $100 \pm 15$. A partir do levantamento do escore de QI, foi possivel fazer uma interpretação qualitativa, na qual acima de 130 pontos de escore é considerado muito superior, 120-129 é superior. 110-119 é médio superior, 90-109 é médio, 80-89 é médio inferior, 70-79 limitrofe e abaixo de 69 é extremamente baixo.

As outras variáveis consideradas neste estudo foram: idade, sexo, ter frequentado pré-escola, frequentar escola no momento da pesquisa, etapa escolar que está frequentando, peso no nascimento, escore de Apgar, dias de internação neonatal, tipo de parto, semanas de gestação, número de consultas no pré-natal, tempo de amamentação exclusivo no seio materno, tempo de amamentação total, histórico de convulsão e desmaio, tabagismo materno na gestação, consumo de álcool ou outras drogas na gestação, histórico de doenças na gravidez, uso de medicação, tabagismo no domicilio durante a gestação, tabagismo atual no domicilio, número de coabitantes na casa, pais morarem juntos na domicilio, tempo de residência na moradia, escolaridade materna, renda familiar e número de irmãos. Essas variáveis foram obtidas através de um questionário no estudo prévio, já citado anteriormente.

Por se tratar de população vulnerável, optou-se por aplicar a escala na própria residência da criança, ao invés de conduzi-la a um posto de saúde ou clínica, evitando, assim, a desistência de participantes e minimizando a intervenção na rotina das familias. As abordagens com a familia, ou responsáveis, foram feitas de junho de 2018 a fevereiro de 2019. Na ocasião, os pesquisadores realizaram a apresentação do estudo, leitura e explicação de Termo de Consentimento Livre e Esclarecido e do Termo de Assentimento, além de, por fim, coleta de dados faltantes no banco de dados pré-existente, referente à história médica pregressa e dados demográficos. Logo após as explicações, sempre a mesma psicóloga aplicava integralmente toda a escala a todas as crianças, para evitar viés nos resultados. Além disso, a escala possui um protocolo de aplicação, que foi seguido pela aplicadora, a fim de evitar não-conformidade e distorções nos resultados.

A participação de todos foi voluntária, os pais ou responsáveis foram informados e assinaram o Termo de Consentimento Livre e Esclarecido e as crianças assinaram o Termo de Assentimento. O estudo foi autorizado pela Secretaria de Saúde de Caxias do Sul, sob o número 477/17, e pelo Comitê de Ética em Pesquisa institucional, com parecer de número 2.599.924.

Considerando que o nivel médio de QI é de 100 , e o desvio padrão é de 15 pontos (17), esperando-se uma diferença de 10 pontos como sendo clinicamente relevantes, com um poder de $90 \% \mathrm{e}$ um alfa de $5 \%$, o tamanho da amostra encontrado foi de 47 crianças em cada grupo. Os modelos para análise dos resultados foram construidos considerando-se todas as variáveis do estudo e as de amamentação dicotomizadas, calculando-se a associação pelo teste do qui-quadrado de Yates. Nos modelos, a variável dependente "QI baixo" foi definida como QI total abaixo de 80.

Para a análise dos grupos de diferentes classificações de QI e as distâncias, foi utilizado o teste de Kruskal-Wallis. Para variáveis preditoras, aplicou-se o método Stepwise Forward não 
condicional, para seleção automática, o qual testa a entrada das variáveis com significância estatística por um escore e as remove, utilizando a probabilidade da função de razão de verossimilhança, baseada na estimativa de máxima verossimilhança parcial. Para as independentes com forte associação entre si, empregou-se no modelo apenas uma delas, aquela com início do fator de risco mais precoce e maior odds ratio não ajustado, em relação à variável dependente. Para selecionar o melhor conjunto de variáveis preditoras, o critério de informação de Akaike's Information Criterion foi usado, que é baseado na máxima verossimilhança. Adotou-se o nivel de significância de 0,05. Após análise de vários modelos de interação, optou-se pelo menor valor de Akaike's Information Criterion. Para análise estatística, foi utilizado o software "R" versão 3.5.2.

\section{Resultados}

Foi verificada uma redução na pontuação do Ql em crianças que habitavam próximas aos sítios de reciclagem, em relação àquelas que viviam a mais de 150 metros de distância, com uma diferença significativa entre as medianas de QI dos dois grupos (Tabela 1). A diferença entre as medianas do QI total dos grupos "exposto" e "não exposto" foi de 11 pontos entre ambos $(p \leq 0,01)$, do Ql verbal foi de 14 pontos $(p \leq 0,01)$ e do Ql de execução foi de 4 pontos $(p=0,04)$.

TABELA 1 - Valores medianos dos escores de quociente de inteligência pela escala Wechsler Abreviada de Inteligência no grupo de expostos $(n=51)$ e controle $(n=49)$.

\begin{tabular}{lccc}
\hline Variáveis & $\begin{array}{c}\text { Expostos } \\
\text { Mediana } \\
\text { (AIQ) }\end{array}$ & $\begin{array}{c}\text { Controles } \\
\text { Mediana } \\
\text { (AIQ) }\end{array}$ & $\mathbf{p}^{*}$ \\
\hline QI verbal & $82(71-92)$ & $96(89-104)$ & $\leq 0,01$ \\
Ql execução & $89(82-92)$ & $93(87-105)$ & 0,04 \\
Ql total & $84(91-73)$ & $95(84-105)$ & $\leq 0,01$ \\
\hline
\end{tabular}

*Teste de Kruskal-Wallis; AIQ: amplitude interquartil; QI: quociente de inteligência; WASI: escala Wechsler Abreviada de Inteligência.

A distância média da residência do local de reciclagem foi de $45 \pm 26 \mathrm{~m}$ no grupo de expostose $354 \pm 175 \mathrm{~m}$ no grupo controle $(p<0,01)$. O tempo de habitação no local, mostrando o tempo de contato da criança com o sítio de reciclagem, não foi estatisticamente diferente entre os dois grupos $(p=0,49)$. Quando avaliada a diferença entre as distâncias de moradia para o local de reciclagem, agrupando-se as crianças em QI médio para cima ( $\geq 90$ ), médio inferior (89-80) e limitrofe/extremamente baixo ( $\leq 80)$, viu-se que a mediana das distâncias foi estatisticamente significativa menor nas crianças de QI baixo e limitrofe ( $p<0,01)$ (Figura 2).

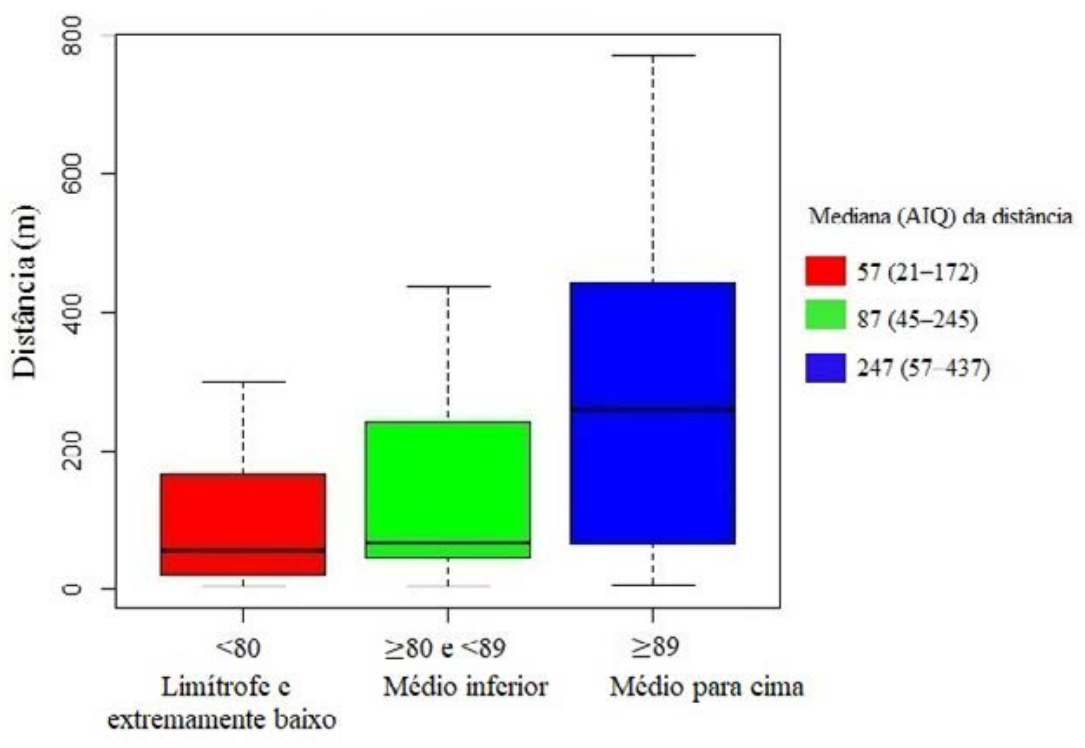

Pontuação de quociente de inteligência

Figura 2 - Classe de quociente de inteligência da amostra e a distância da moradia. 
O estudo contou com 100 participantes, sendo 50 do gênero masculino. Embora tenha havido diferença significativa entre os grupos das variáveis peso de nascimento, escolaridade materna e de renda familiar, a amostra, de um modo geral, se caracterizou por uma população de baixa renda e baixa escolaridade materna. No entanto, não houve diferença entre os grupos quanto a indicadores de precariedade no cuidado perinatal que poderiam conferir maior vulnerabilidade precoce ao neurodesenvolvimento. A população total estudada conta com baixa escolaridade materna, sendo que 58\% das mães têm apenas o ensino fundamental in- completo. Do mesmo modo, a renda, variável usada como outro marcador da condição socioeconômica, foi baixa, com 30\% da população vivendo com menos de mil reais por mês. Esse dado, juntamente com a escolaridade materna, foi posteriormente incluída no modelo de regressão logística.

Quanto às demais variáveis com potencial de influenciar o neurodesenvolvimento, não houve diferença significativa entre os grupos, à exceção do número de coabitantes e o tabagismo atual no domicílio, que foram maiores no grupo de crianças expostas à reciclagem (Tabela $\mathbf{2}$ ).

TABELA 2 - Características das crianças dos grupos de expostos (habitação a menos de 100 metros da reciclagem) e do grupo controle (mais de 150 metros).

\begin{tabular}{|c|c|c|c|}
\hline Variáveis & Expostos $(n=51)$ & Controle $(n=49)$ & $\mathbf{p}$ \\
\hline Idade, anos* & $9,5 \pm 2,1$ & $9,8 \pm 2,2$ & $0,15^{\ddagger}$ \\
\hline Peso nascimento, $\mathrm{g}^{*}$ & $3.214 \pm 447$ & $3.035 \pm 430$ & $<0,01^{\ddagger}$ \\
\hline Escore de Apgar no $5^{\circ}$ minuto ${ }^{+}$ & $9(8-10)$ & $9(8-10)$ & $0,8^{\ddagger}$ \\
\hline Dias de Internação pós-natal ${ }^{\dagger}$ & $2(1-3)$ & $2(1-2)$ & $0,6^{5}$ \\
\hline Tabagismo materno na gestação, n(\%) & $18(35,3)$ & $12(24,5)$ & $0,6^{5}$ \\
\hline Consumo de álcool na gestação, n(\%) & $5(9,8)$ & $6(12,2)$ & $0,5^{\S}$ \\
\hline Doenças/problemas na gestação, n(\%) & $14(27,5)$ & $12(24,5)$ & $0,1^{5}$ \\
\hline Escolaridade materna $<8$ anos, n(\%) & $28(55)$ & $9(18,4)$ & $<0,01^{\S}$ \\
\hline Renda familiar mensal, $\mathrm{R} \$^{+}$ & $1100(569-1631)$ & $2500(1175-3825)$ & $<0,01^{\ddagger}$ \\
\hline Criança frequentou pré-escola, n(\%) & $37(72,5)$ & $31(63)$ & $0,4^{5}$ \\
\hline Amamentação exclusiva $\geq 4$ meses, $n(\%)$ & $23(45)$ & $29(59)$ & $0,1^{\S}$ \\
\hline Mora com ambos os pais, $n(\%)$ & $29(57)$ & $30(61)$ & $0,08^{5}$ \\
\hline Tempo de residência, em meses ${ }^{*}$ & $148 \pm 86$ & $137 \pm 80$ & $0,5^{\ddagger}$ \\
\hline $\mathrm{N}^{\circ}$ de coabitantes ${ }^{\dagger}$ & $5(4-6)$ & $4(3-4)$ & $<0,01^{5}$ \\
\hline$N^{\circ}$ de irmãos ${ }^{\dagger}$ & $3(1-5)$ & $1(2)$ & $0,02^{\ddagger}$ \\
\hline Tabagismo atual no domicilio, n(\%) & $30(59)$ & $16(33)$ & $<0,01^{\S}$ \\
\hline
\end{tabular}

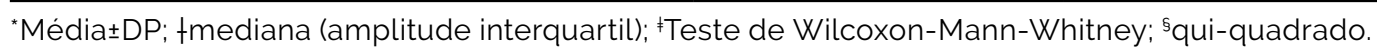

Em relação aos aspectos socioeconômicos da amostra, muitas variáveis mostraram dados relevantes à caracterização da população estudada, mesmo que não tenha havido diferenças significativas entre os grupos de expostos e de controle. De toda a amostra, 32\% não tiveram acesso à pré- -escola. Outros aspectos habitualmente relevantes ao neurodesenvolvimento também se mostraram desfavoráveis às crianças: 52\% delas foram amamentadas exclusivamente no seio materno, por pelo menos quatro meses; $30 \%$ dos bebês tiveram mães que fumaram durante a gestação; 11\% com 
mães que consumiram álcool durante a gestação; 26\% das mães tiveram doenças ou problemas na gestação; 41\% não moram mais com ambos os pais no domicilio, conforme mostra a Tabela 2.

Optou-se por colocar no modelo de análise multivariada as covariáveis renda familiar, distância menor de $100 \mathrm{~m}$ do sítio da reciclagem, alta escolaridade materna, ter ou não frequentado pré-escola e morar com ambos os pais (Tabela 3).

TABELA 3 - Análise simples e ajustada das covariáveis para quociente de inteligência abaixo do normal.

\begin{tabular}{llll}
\hline \multicolumn{1}{c}{ Covariáveis } & \multicolumn{1}{c}{$\begin{array}{c}\text { OR não-ajustado } \\
\text { (IC 95\%) }\end{array}$} & $\begin{array}{c}\text { OR ajustado } \\
\text { (IC 95\%) }\end{array}$ & $\mathbf{p}^{*}$ \\
\hline Baixa renda familiar & $3,29(1,41-7,66)$ & $2,22(0,75-6,57)$ & 0,15 \\
Distância $<100 \mathrm{~m}$ da reciclagem & $2,19(1,21-3,95)$ & $2,25(0,83-6,13)$ & 0,1 \\
Escolaridade materna $\geq 8$ anos & $0,13(0,05-0,35)$ & $0,28(0,11-0,72)$ & $<0,01$ \\
Frequentar pré-escola & $0,68(0,29-1,6)$ & $0,72(0,27-1,91)$ & 0,5 \\
Mora com ambos os pais & $0,49(0,22-1,1)$ & $0,55(0,2-1,47)$ & 0,2 \\
\hline
\end{tabular}

*Teste de Wald; QI: quociente de inteligência.

Quanto aos fatores de risco com potencial de afetar o neurodesenvolvimento, a distância menor do que 100 m se mostrou estatisticamente significativa na análise do odds ratio não ajustado, no entanto, quando controladas as diferenças, o odds ratio ajustado não mostrou ser estatisticamente significativo. A alta escolaridade materna mostrou-se como um fator protetor, ou seja, uma mãe com alta escolaridade reduz em $72 \%$ a chance do filho ter um QI baixo, independentemente de qualquer outro fator (Figura 3).

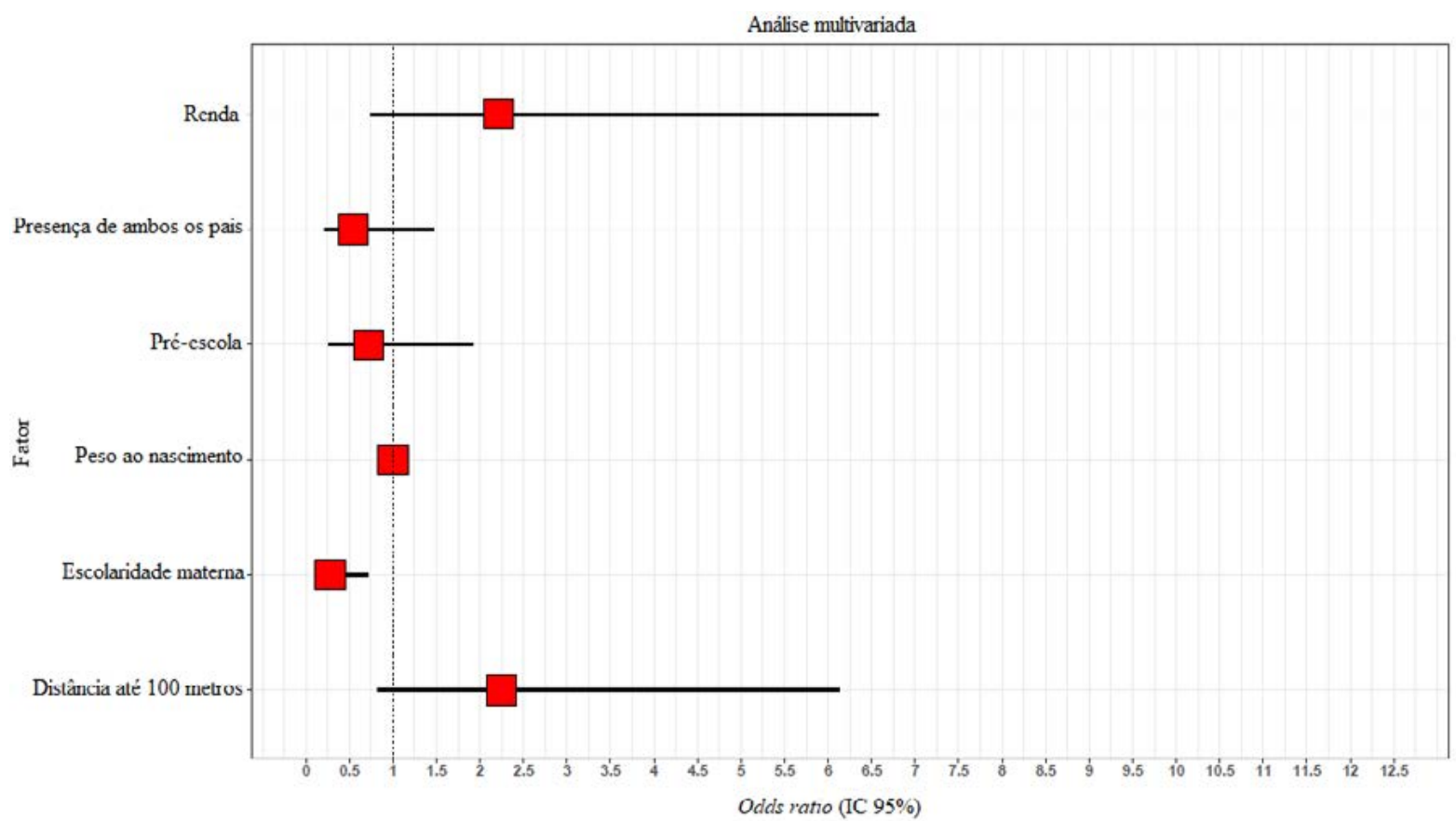

Figura 3 - Odds ratio ajustado das covariáveis com impacto no quociente de inteligência. 


\section{Discussão}

Neste estudo, foi demonstrada uma associação entre a habitação próxima a sítios de reciclagem e menor desempenho na escala de avaliação de QI verbal, QI execução e QI total em escolares, mesmo com o controle de outras variáveis com potencial de atraso do neurodesenvolvimento. Até o presente, os autores desconhecem outros estudos avaliando especificamente essa associação. Há estudos que mostram a proximidade ao aterro sanitário como um fator de prejuizo ao neurodesenvolvimento (18), além da publicação que investigou a associação entre exposição a resíduos urbanos e dificuldades emocionais e comportamentais de escolares (15). Outros estudos têm focado nos sítios de descarte do chamado "lixo eletrônico", ou "e-waste" (10), mostrando a associação entre a habitação de crianças próximas a lugares dedicados a esse tipo de atividade e niveis séricos elevados de chumbo (11), assim como outros metais, em amostras de cabelo $(12,13,19,20)$ e, até mesmo, nos alimentos (21). Ainda, há outras publicações que investigam a associação entre exposição a metais especificos e habilidades cognitivas $(1,3,8,9,11-13,19,20)$. Contudo, esses estudos diferem do presente na medida em que os resíduos encontrados nesta pesquisa eram constituidos de forma mista, com matéria orgânica, produtos químicos e metais sem separação prévia.

Comparando os resultados de algumas dessas pesquisas, que utilizaram alguma escala de QI, com os resultados encontrados neste presente estudo, observa-se que o QI tende a reduzir quanto mais próximo da exposição aos locais de reciclagem, assim como o Ql é menor nos estudos onde há maior concentração de chumbo $(3,11)$, arsênico (12), cadmio (19), fluoreto (9) e manganês (13) no sangue das crianças, ou até mesmo nos quais se mediu o Ql de jovens que moravam próximo a depósitos de residuos sólidos tóxicos (20).

O critério de corte das distâncias em 100 metros baseou-se no usado na maioria dos estudos que avaliam poluição do ar (22-24), uma vez que não existe, no conhecimento dos autores, uma pesquisa pregressa, com pontos de corte bem estabelecidos para avaliar o impacto da proximidade dos sítios de reciclagem de resíduos mistos com habilidades cognitivas. Sendo assim, no modo como o presente estudo foi desenhado, residir a uma distância menor de 100 metros do sítio de reciclagem resulta em QI baixo apenas na análise de odds ratio não ajustado. Entende-se que, se houvesse um número maior de crianças participantes, controlando-se as covariáveis com impacto no QI, a tendência seria que o fator "distância menor de 100 metros" sustentaria a significância estatística também no odds ratio ajustado.

A Organização Mundial da Saúde considera necessários mais estudos avaliando exposições ambientais em crianças de países como os da América Latina, para, assim, realizar, não só diagnóstico, mas também auxiliar na adoção de politicas públicas (25). Países em desenvolvimento, como o Brasil, sofrem dupla carga ambiental, uma vez que recebem agressões ambientais relacionadas à industrialização - com residuos característicos e, ao mesmo tempo, sofrem as agressões ambientais advindas da pobreza e da falta de educação ambiental (26). No presente estudo, tanto a população do grupo exposto quanto a do grupo controle mostravam baixa renda, baixa escolaridade materna e um terço de crianças não frequentou a pré-escola, caracterizando, dessa forma, a vulnerabilidade social e ambiental, que pode impactar no desenvolvimento cognitivo, como postulado pelo modelo teórico do estudo Adverse Childhood Experiences, e que foi o modelo adotado para este estudo (27, 28). No entanto, dentre os resultados da análise multivariada, foi possivel observar que a maior escolaridade materna foi um fator que contribuiu para um QI maior. Esses achados permitem elucubrar que, mesmo que a moradia esteja a menos de 100 metros do sítio de reciclagem, o efeito do QI baixo talvez não esteja ligado a questões inalatórias das crianças, mas, sim, dérmicas ou de ingestão. Ou seja, se a mãe tem conhecimento, zela pela saúde dos filhos, e solicita para que não manipulem resíduos orgânicos (dérmica) 
e nem levem as mãos sujas à boca (ingestão), isso porque a mãe tem maior percepção de risco ambiental. Se fosse uma questão inalatória, de ar contaminado, a intervenção da mãe não teria efeito, porque todos precisam respirar. Sendo assim, a educação ambiental, além da formal, possa, talvez, ser efetiva na mitigação do impacto ambiental. Isso reforça a importância de programas destinados à população com maior vulnerabilidade. É possivel que essa educação seja feita ainda no pré-natal, antes da exposição, como sugere Heckman et al. (29).

O tabagismo atual no domicílio foi uma covariável que apresentou uma diferença significativa entre os grupos de expostos e controle. Analisando alguns estudos que versam sobre tabagismo e neurodesenvolvimento da criança, entende-se que há uma associação entre tabagismo na gestação e também tabagismo atual no domicilio ao Ql eà performance escolar das crianças, podendo ter uma queda no $\mathrm{Q}$ l de até 6,85 pontos naqueles cujos pais fumam em casa (30).

Um aspecto que merece destaque nesta análise foi a abordagem dada à caracterização da exposição. Optou-se por adotar a teoria do "expossoma", ao invés de utilizar-se de biomarcadores, uma vez que as exposições variam ao longo do tempo. Segundo essa teoria, as inúmeras agressões ambientais podem atuar de maneira sinérgica para um determinado desfecho. Dessa forma, evita-se que a busca, eventualmente fracassada, por um poluente específico possa conduzir à conclusão de ausência de outros poluentes e baixa carga ambiental sobre as crianças (14). As publicações que buscaram comparar o QI com a exposição a um poluente especíico $(3,4,9,12,19)$ tiveram uma redução menor do QI do que foi encontrado neste estudo. Baseando-se nessa teoria, é mais coerente avaliar a exposição aos resíduos como um todo, ao invés de buscar um dentre milhares especificos possiveis de serem (ou não) encontrados. Ao destacar a distância entre a casa da criança e o sítio de reciclagem, a abordagem desta pesquisa joga o foco sobre a rotina da criança, ao invés de se limitar a um marcador específico.
O melhor desempenho no grupo de expostos foi o Ql execução, que é a capacidade fluída da inteligência, rapidez e exatidão do raciocínio abstrato, especialmente para resolução de novos problemas. Se compararmos esse resultado, com os estudos de populações vulneráveis, envolvendo crianças e adolescentes que vivem em situações de rua, encontra-se uma explicação de que sobreviver e vivenciar as experiências na rua implica em criação e exercícios de estratégias variadas, permitindo o desenvolvimento de diversas habilidades e formas de raciocínio (31). A chamada "sabedoria de rua" advém de viver o dia a dia na rua, que envolve a aprendizagem da sobrevivência nesse espaço, a aquisição e utilização de repertórios comportamentais especíicos para cada situação e contato social (31). Além disso, pensa-se que o uso criativo de objetos encontrados na rua (ou, nesse caso, nos sítios de reciclagem), e que não são usualmente identificados como brinquedos, estimulam a criação e a realização de atividades lúdicas. O processo de brincar na rua valoriza habilidades e conquistas das crianças nos aspectos sociocognitivos envolvidos, ou seja, as formas alternativas de brinquedos e brincadeiras permitem que se mantenham à revelia das condições socioeconômicas e estimulam o desenvolvimento cognitivo (32).

Partindo-se do pressuposto de que a média do QI no Brasil é de 100 pontos de escore (17), tanto o grupo de expostos quanto o grupo controle estão com as médias abaixo da brasileira. O melhor desempenho do grupo controle ficou no QI verbal, com quatro pontos abaixo da média, e a melhor pontuação do grupo de expostos foi de 11 pontos abaixo da média. Isso é preocupante, tanto do ponto de vista social quanto educacional. Estudos mostram o prejuizo coletivo e populacional grave quando se tem uma redução de 5 pontos no QI $(3,12,19)$. A perda de cinco pontos no QI pode não afetar a capacidade de um individuo de viver uma vida produtiva, mas, se essa perda é vivida por uma população inteira, as implicações para a sociedade podem ser profundas. Um estudo examinou o impacto social da aparente pequena 
diminuição na inteligência. Por exemplo, uma população afetada de 260 milhões de pessoas (como nos Estados Unidos da América), com uma média de QI de 100 e um desvio padrão de 15 , haveria seis milhões de pessoas com Ql acima de 130 e seis milhões abaixo de 70. Uma diminuição na média de QI de cinco pontos mudaria a distribuição para a esquerda, ou seja, o número de pessoas de pontuação acima de 130 cairia 3,6 milhões, enquanto o número abaixo de 70 aumentaria em 3.4 milhões (33).

Os dados apresentados apontam para uma associação entre a proximidade a sítios de reciclagem e a diminuição no QI. Sendo assim, parece razoável, a partir dos resultados apresentados, ao menos colocar o problema na perspectiva dos profissionais de saúde pública e dos gestores da saúde e do meio ambiente.

Este estudo apresentou algumas limitações. Talvez o maior limitador tenha relação com o local onde se aplicou a escala. Segundo o protocolo de testagem, é permitida a aplicação na residência, porém, esta deve estar livre de distrações ou interrupções externas, ter boa iluminação, ventilação e, caso tenha algum acompanhante presente, este deve ficar atrás do examinando, sem influenciar nas respostas (17). Em função do cuidado ético dos autores em evitar ao máximo interferir na rotina familiar das crianças estudadas, as interrupções não puderam ser totalmente neutralizadas (Figura 4).

Outra limitação é a não comprovação da exposição ambiental através de biomarcadores. No entanto, ao se optar pela abordagem do expossoma, esse tipo de caracterização torna-se menos relevante, pois pode negligenciar exposições não cobertas pelo biomarcador ou espécie-sentinela.

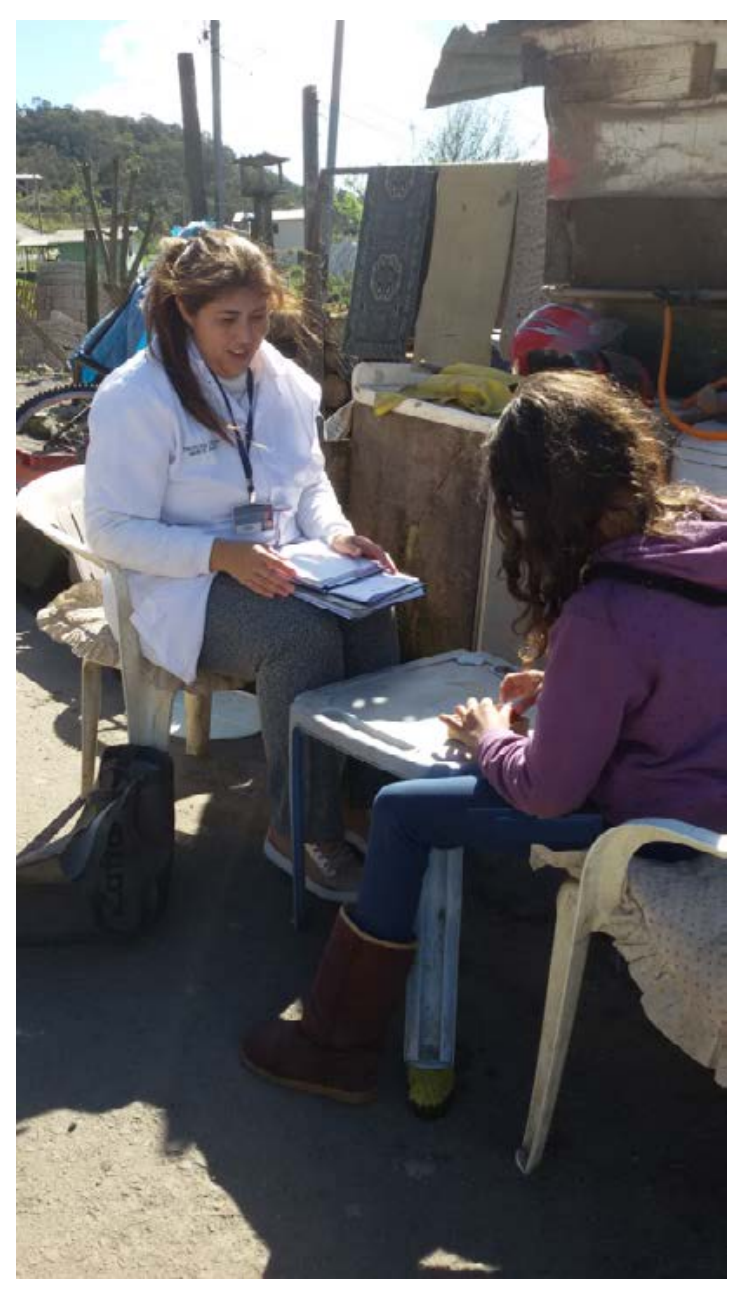

Figura 4 - Improvisando mobília no local para aplicação da escala.

É imprescindivel destacar a presença de outros fatores associados às más condições socioeconômicas, que poderiam ser confusos neste estudo. Apesar do controle, através da análise estatística adotada, é possivel que outros fatores sejam analisados em estudos futuros. De qualquer modo, a análise aborda um problema negligenciado em centros urbanos, que é a segurança sanitária e os riscos à saúde, colocados pela exposição de crianças a residuos. Até o presente momento, os autores desconhecem estudos semelhantes, com a combinação de georeferenciamento e o uso de instrumento validado, como a escala WASI.

A partir desta pesquisa apresentada, conclui-se que a distância entre a habitação e os sítios de reciclagem de residuos pode reduzir habilidades cognitivas em escolares, e esse efeito parece ser atenuado pela escolaridade materna. Essa associação reforça as preocupações sobre o 
impacto do manejo inadequado de resíduos urbanos, podendo vir a subsidiar a criação de políticas públicas que visem diminuir exposições ambientais potencialmente danosas.

É necessária a atenção dos Stakeholders, para detectar todas as variáveis que interferem no neurodesenvolvimento das crianças, criando ferramentas, tecnologias ou dispositivos que possam evitar desfechos danosos, tanto do ponto de vista individual para o ser humano afetado quanto do ponto de vista de sociedade, e que também se possa agir com ações de prevenção.

Tanto a educação formal quanto a educação ambiental devem ser incluídas nas políticas públicas como forma de prevenção, com vias à saúde global do ser humano e do planeta que habita.

\section{Notas}

Este estudo é parte do resultado de dissertação de mestrado do Programa de Pós-Graduação em Ciências da Saúde da Universidade de Caxias do Sul de uma das autoras (LCMB), intitulada "Efeito da distância entre habitação e sítios de reciclagem de resíduos sobre habilidades cognitivas em escolares", que foi apresentado no $39^{\circ}$ Congresso Brasileiro de Pediatria, promovido pela Sociedade Brasileira de Pediatria, ocorrido de 9 a 12 de outubro de 2019, em Porto Alegre, RS, Brasil.

\section{Apoio financeiro}

O presente trabalho foi realizado com apoio da Coordenação de Aperfeiçoamento de Pessoal de Nivel Superior (CAPES) - código de financiamento 001.

Um dos autores (DA) foi apoiado pelo Conselho Nacional de Desenvolvimento Científico e Tecnológico (CNPq).

\section{Declaração de conflito de interesses}

Os autores declaram não haver conflitos de interesses relevantes ao conteúdo deste estudo.

\section{Contribuições dos autores}

Todos os autores fizeram contribuições substanciais para concepção, ou delineamento, ou aquisição, ou análise ou interpretação de dados; e redação do trabalho ou revisão crítica; e aprovação final da versão para publicação.

\section{Disponibilidade dos dados e responsabilidade pelos resultados}

Todos os autores declaram ter tido total acesso aos dados obtidos e assumem completa responsabilidade pela integridade destes resultados.

\section{Referências}

1. Beth KS. CDC updates guidelines for children's lead exposure. Environmental Health Perspectives [Internet]. 2012 [citado $2020 \mathrm{Jul}$ 30];120(7):A268. Disponivel em: https://link.gale.com/apps/doc/A304467277/ AONE?u=capes\&sid=AONE\&xid=a930ba1a

2. Grossman E. High Tech trash: digital devices, hidden toxins and human health. Washington (DC): Island Press; 2006. $334 \mathrm{p}$

3. Liu J, Li L, Wang Y, Yan C, Liu X. Impact of low blood lead concentrations on IQ and school performance in chinese children. PloS One. 2013; 8(5):e65230. https:// doi.org/10.1371/journal.pone.0065230

4. Rodríguez-Barranco M, Lacasaña M, Aguilar-Garduño C, Alguacil J, Gil F, González-Alzaga B, et al. Association of arsenic, cadmium and manganese exposure with neurodevelopment and behavioural disorders in children: A systematic review and meta-analysis. Science of The Total Environment. 2013; 454-55:562-77. https:// doi.org/10.1016/j.scitotenv.2013.03.047

5. Lam J, Lanphear BP, Bellinger D, Axelrad DA, McPartland J, Sutton P, et al. Developmental PBDE Exposure and IQ/ADHD in childhood: a systematic review and meta-analysis. Environmental health perspectives. 2017; 125(8):86001. https://doi.org/10.1289\%2FEHP1632

6. World Health Organization. Principles for evaluating health risks in children associated with exposure to chemicals. Enviromental health criteria 237 [Internet]. Geneva: World Heath Organization; 2006 [Citado 2020 Jul 30]. Disponivel em: https://apps.who.int/iris/handle/10665/43604

7. Landrigan PJ, Goldman LR. Children's vulnerability to toxic chemicals: a challenge and opportunity to strengthen health and environmental policy. Health Aff (Millwood) 2011; 30(5):842-50. https://doi.org/10.1377/hlthaff.2011.0151

8. Chen A, Dietrich KN, Huo X, Ho S. Developmental neurotoxicants in e-waste: an emerging health concern Environ Health Perspect. 2011; 119(4):431-8. https://doi. org/10.1289/ehp.1002452

9. Bashash M, Thomas D, Hu H, Martinez-Mier EA, Sanchez BN, Basu N, et al. Prenatal Fluoride Exposure and Cognitive Outcomes in Children at 4 and 6-12 Years of Age in Mexico. Environ Health Perspect. 2017: 125(9):97017. https://doi.org/10.1289/ehp655 
10. Grant K, Goldizen FC, Sly PD, Brune MN, Neira M, van den Berg $M$, et al. Health consequences of exposure to e-waste: a systematic review. Lancet Glob Health. 2013; 1(6):e350-61. https://doi.org/10.1016/ s2214-109x(13)70101-3

11. Tongesayi T, Kugara J, Tongesayi S. Waste dumpsites and public health: a case for lead exposure in Zimbabwe and potential global implications. Environ Geochem Health. 2018; 4O(1):375-81. https://doi.org/10.1007/s10653-017-9917-6

12. Wright $R O$, Amarasiriwardena $C$, Woolf $A D$, Jim $R$, Bellinger DC. Neuropsychological correlates of hair arsenic, manganese, and cadmium levels in school-age children residing near a hazardous waste site. Neurotoxicology. 2006; 27(2):210-6. https://doi.org/10.1016/j. neuro.2005.10.001

13. Riojas-Rodriguez $H$, Solis-Vivanco R, Schilmann A, Montes S, Rodríguez S, Ríos C, et al. Intellectual function in Mexican children living in a mining area and environmentally exposed to manganese. Environ Health Perspect. 2010; 118(10):1465-70. https://doi. org/10.1289/ehp.0901229

14. Renz H, Holt PG, Inouye M, Logan AC, Prescott SL, Sly PD. An exposome perspective: Early-life events and immune development in a changing world. The J Allergy Clin Immunol. 2017; 140(1):24-40. https://doi. org/10.1016/j.jaci.2017.05.015

15. Dumcke TS, Benedetti A, Selistre LSD, Camardelo AMP, Silva ERD. Association between exposure to urban waste and emotional and behavioral difficulties in schoolchildren. J Pediatr (Rio J). 2020; 96(3):364-70. https://doi.org/10.1016/j.jped.2018.11.014

16. Colvero DA, Gomes APD, Tarelho LADC, Matos MAAD, Santos KAD. Use of a geographic information system to find areas for locating of municipal solid waste management facilities. Waste Management. 2018; 77:500-15. https://doi.org/10.1016/j.wasman.2018.04.036

17. Wechsler D. Escala Wechsler Abreviada de Inteligência: WASI . 1ªd. São Paulo: Casa do Psicólogo; 2014.

18. Sarigiannis DA. Assessing the impact of hazardous waste on children's health: The exposome paradigm. Environ Res. 2017; 158:531-41. https://doi.org/10.1016/j. envres.2017.06.031

19. Rodríguez-Barranco M, Lacasaña M, Gil F, Lorca A, Alguacil J, Rohlman DS, et al. Cadmium exposure and neuropsychological development in school children in southwestern Spain. Environ Res. 2014; 134:66-73. https://doi.org/10.1016/j.envres.2014.06.026

20. Burgos S, Tenorio M, Zapata P, Cáceres DD, Klarian J, Alvarez N, et al. Cognitive performance among cohorts of children exposed to a waste disposal site containing heavy metals in Chile. Int J Environ Health Res. 2017; 27(2):117-25. https://doi.org/10.1080/09603123.2017.12924.94

21. Zheng J, Chen K, Yan X, Chen SJ, Hu GC, Peng XW, et al. Heavy metals in food, house dust, and water from an e-waste recycling area in South China and the potential risk to human health. Ecotoxicology and Environmental Safety. 2013; 96:205-12. https://doi. org/10.1016/j.ecoenv.2013.06.017
22. Cook AG, deVos AJBM, Pereira G, Jardine A, Weinstein $P$. Use of a total traffic count metric to investigate the impact of roadways on asthma severity: a case-control study. Environ Health. 2011; 10(1):52. https:// doi.org/10.1186/1476-069X-10-52

23. Cesaroni G, Badaloni C, Romano V, Donato E, Perucci $\mathrm{CA}$, Forastiere F. Socioeconomic position and health status of people who live near busy roads: the Rome Longitudinal Study (RoLS). Environ Health. 2010; 9(1):41. https://doi.org/10.1186/1476-069X-9-41

24. Harris MH, Gold DR, Rifas-Shiman SL, Melly SJ, Zanobetti A, Coull BA, et al. Prenatal and Childhood traffic-related pollution exposure and childhood cognition in the Project Viva cohort (Massachusetts, USA). Environ Health Perspect. 2019; 127(10):1072-8. https:// doi.org/10.128.9/ehp.1408803

25. Laborde A, Tomasina F, Bianchi F, Bruné M-N, Buka I. Comba P, et al. Children's health in Latin America: the influence of environmental exposures. Environ Health Perspect. 2015; 123(3):201-9. https://doi.org/10.1289/ ehp.1408292

26. Suk WA, Ahanchian H, Asante KA, Carpenter DO, Diaz-Barriga $\mathrm{F}, \mathrm{Ha} \mathrm{EH}$, et al. Environmental pollution: an under-recognized threat to children's health, especially in low- and middle-income countries. Environ Health Perspect. 2016; 124(3):A41-5. https://doi.org/10.1289/ ehp.1510517

27. Harris NB. The Deepest well: healing the long-term effects of childhood adversity. New York: Houghton Mifflin Harcourt; 2018.

28. Felitti VJ, Anda RF, Nordenberg D, Williamson DF Spitz AM, Edwards V, et al. Relationship of childhood abuse and household dysfunction to many of the leading causes of death in adults. The Adverse Childhood Experiences (ACE) Study. Am J Prev Med. 1998; 14(4):24558. https://doi.org/10.1016/s0749-3797(98)00017-8

29. Doyle O, Harmon CP, Heckman JJ, Tremblay RE. Investing in early human development: timing and economic efficiency. Econ Human Biol. 2009; 7(1):1-6. https://doi.org/10.1016/j.ehb.2009.01.002

30. Herrmann M, King K, Weitzman M. Prenatal tobacco smoke and postnatal secondhand smoke exposure and child neurodevelopment. Curr Opin Pediatr. 2008; 20(2):184-90. https://doi.org/10.1097/ mop.ob013e3282f56165

31. Alves PB, Koller SH, Silva AS, Santos CL do, Silva MR da, Reppold CT, et al. Atividades cotidianas de crianças em situação de rua. Psic Teor Pesq. 2002; 18(3):305-13. https://doi.org/10.1590/S0102-37722002000300010

32. Pontes FAR, Magalhães CMC. A estrutura da brincadeira e a regulação das relações. Psic Teor Pesq. 2002; 18(2):213-9. https://doi.org/10.1590/S010237722002000200011

33. Stein J, Schettler T, Wallinga D, Valenti M. In harm's way: toxic threats to child development. J Dev Behav Pediatr. 2002; 23(1 Suppl):S13-22. https://doi. org/10.1097/00004703-200202001-00004 


\section{Luciana Cristina Mancio Balico}

Doutoranda em Ciências da Saúde na Universidade de Caxias do Sul. Psicóloga Clínica em Caxias do Sul, RS, Brasil.

\section{Diane Arbusti}

Médica graduada pela Universidade de Caxias do Sul. Residente do Serviço de Pediatria do Hospital da Criança Conceição em Porto Alegre, RS, Brasil.

\section{Luciano da Silva Selistre}

Pós-doutorado em Bioestatística, Biometria e Biologia Evolutiva. Professor do Curso de Medicina e do Programa de Pós-Graduação em Ciências da Saúde na Universidade de Caxias do Sul, RS, Brasil.

\section{Ana Maria Paim Camardelo}

Doutora em Serviço Social pela Pontificia Universidade Católica do Rio Grande do Sul. Professora do Curso de Serviço Social, dos Programas de Pós-Graduação em Direito e em Psicologia da Universidade de Caxias do Sul, RS, Brasil.

\section{Emerson Rodrigues da Silva}

Doutor em Saúde da Criança e do Adolescente pela Universidade de Campinas. Professor do Curso de Medicina e do Programa de Pós-Graduação em Ciências da Saúde da Universidade de Caxias do Sul, RS, Brasil.

\section{Endereço para correspondência}

Luciana Cristina Mancio Balico

Universidade de Caxias do Sul

Rua Francisco Getúlio Vargas, 1130

Petrópolis, 95070-560

Caxias do Sul, RS, Brasil

Os textos deste artigo foram revisados pela Zeppelini Publishers e submetidos para validação do(s) autor(es) antes da publicação. 